\title{
TAP-ing into TIEPPs for cancer immunotherapy
}

\author{
Rolf Kiessling
}

Department of Oncology and Pathology, Cancer Center Karolinska, Karolinska Institutet, Stockholm, Sweden.

\begin{abstract}
Cancer immunotherapy in which cytotoxic T cells (CTLs) target tumorspecific antigens complexed to MHC-I molecules has been used successfully for several types of cancer; however, MHC-I is frequently downregulated in tumors, resulting in CTL evasion. Recently, it has been shown that MHC-I $\mathrm{I}^{10}$ tumors produce a set of T cell epitopes associated with impaired peptide processing (TEIPP) that have potential to be exploited for immunotherapy. TEIPP-specific CTLs recognize tumors defective in antigen presentation machinery (APM) but not those with intact APM. In this issue of the $J C I$, Doorduljn et al. evaluated thymus selection and peripheral behavior of TEIPP-specific T cells, using a unique T cell receptor (TCR) transgenic mouse model. They demonstrated that TEIPP-specific T cells in TAP-deficient mice have largely been deleted by central tolerance, while the same T cells in WT mice are naive and sustained. The results of this study suggest that TIEPPs have potential to be successful targets for elimination of MHC-I ${ }^{10}$ tumors.
\end{abstract}

with intact APM. Previously, van Hall and colleagues identified the target of these $\mathrm{T}$ cells as a class of peptides that only appears in tumor cells with defective APM and therefore has been termed TEIPP ( $\mathrm{T}$ cell epitopes associated with impaired peptide processing) (7). Although TEIPPs are preferentially expressed on tumors with defective APM, the recognition of these peptides is dependent on residual MHC-I and $\beta-2$ microglobulin on the targets (7). A diverse pool of CTL clones with distinct $\mathrm{MHC}$ restriction patterns recognize TAP-deficient cells, predominantly in context of the nonclassical MHC molecule Qa-1 b $(7,8)$.

\section{Characterization of TEIPP-specific T cells}

TEIPPs are derived from ubiquitous nonmutated proteins and therefore belong to the group of so-called tumor self-antigens. These antigens are overexpressed in cancer cells compared with normal tissues, thereby providing a window for therapeutic intervention. The efficacy of targeting these normal proteins remains unproven, probably as the immune system has been anergized through the process of central tolerance in the thymus. Furthermore, when this tolerance is overcome, the "selfness" and expression of these proteins in normal tissues constitutes a risk for serious side effects, as has been observed recently $(9,10)$. The ingeniousness of targeting TEIPPs lies in that central tolerance to these epitopes is not operational, as the normal thymus epithelium possesses a functional APM and fully operative TAP molecules. Accordingly, thymic epithelial cells potentially do not present TEIPP epitopes, and as TEIPP-recognizing $\mathrm{T}$ cells are not subject to central tolerance, they share a lack of tolerance induction with genuinely foreign antigens, including those derived from microbes and neoantigens derived from somatic tumor mutations. Yet, what sets TEIPPs apart from tumor-derived neoantigens is that they are derived from normal, nonmutated, ubiquitously expressed, germline-encoded housekeeping proteins. 
In this issue, Doorduljn et al. (11) have studied the potential autoimmunity that may develop with TEIPP-based immunotherapy by constructing a T cell receptor-transgenic (TCR-tg) mouse model bearing a MHC-I-restricted $\left(\mathrm{H}-2 \mathrm{D}^{\mathrm{b}}\right) \mathrm{TCR}$ against the prototypic TEIPP antigen TRH4. Using this model, Doorduljn and colleagues have studied thymic selection and function of TEIPP-specific T cells in a way that has hitherto not been possible. As expected from other transgenic models of TCRs specific for MHC-I-restricted epitopes, a strong bias toward peripheral $\mathrm{CD}^{+}$cells was observed in this TCR-tg model. The hypothesis that TEIPP-specific $\mathrm{T}$ cells are not being exposed to TEIPP epitopes in the thymic epithelium is supported by the naive phenotype of the $\mathrm{CD}^{+} \mathrm{T}$ cells in transgenic mice, which is comparable to T cells from WT mice. Crossing TCR-tg mice with TAP1-deficient mice confirmed earlier observations that thymic epithelium expresses the Trh4 gene and has the potential to present the TRH4/H-2 complex when rendered TAP deficient. Predictably, the thymus of TAP-deficient TCR-tg mice lacked mature $\mathrm{CD}^{+} \mathrm{T}$ cells and had a reduced population of double-positive $\mathrm{T}$ cells and thymic cellularity. Cumulatively, these data indicate that the TEIPP-specific $\mathrm{T}$ cells in TAP-deficient mice have largely been deleted, while the same T cells in WT mice are naive and sustained.

\section{Conclusions and future directions}

The observations made by Doorduljn et al. have implications for the potential use of TEIPP epitopes for cancer immunotherapy. Specifically, the key to more efficient immunotherapies is the induction of tumor-specific $\mathrm{T}$ cells that have not undergone central tolerance and therefore have a high affinity for and are highly likely to eliminate tumor cells. A great deal of effort is directed at targeting mutant neoantigens derived from somatic point mutations in cancer cells (12). Are TEIPP epitopes an alternative to mutated neoepitopes, as $\mathrm{T}$ cells targeting both of these antigens have not undergone central tolerance? Mutant neoantigens are laborious and time consuming to define, as their identification requires whole-exome sequencing and extensive RNA expression and bioinfor- matic analysis. In contrast, TEIPP epitopes are broadly expressed on antigen-deficient tumor cells and can be considered "on the shelf" to utilize as tumor vaccines in a manner similar to tumor differentiation or cancer testis antigens; however, TEIPPs are likely to possess a much greater $\mathrm{T}$ cell activation potential.

Our knowledge of TEIPP antigens, however, is presently restricted to the prototype TRH4 antigen, and the extent to which this antigen is analogous to other TEIPP antigens remains unknown. As attractive as TEIPP-based therapies may seem, they are directed against self-proteins and carry a tangible risk of inducing on-target autoimmune side effects. These concerns are appropriate, as serious adverse events resulting from targeting overexpressed, nonmutated differentiation antigens have been recently reported (9, 10). Doorduljn et al. address these concerns and demonstrated that TEIPPspecific $\mathrm{T}$ cells are innocuous when transferred into syngeneic B6 mice - even in the presence of inflammatory signals induced by agonistic anti-CD40 antibody (11). As a control, transfer of $\mathrm{T}$ cells into TAP-deficient mice, which express TEIPP epitopes, resulted in clear signs of antigen activation. These experiments are reassuring; however, the TEIPP epitope repertoire may be extensive, and experiments targeting one single prototype TEIPP epitope may have limited value.

It is also possible that immunotherapy directed at APM-deficient cells can activate $\mathrm{T}$ cells specific for epitopes expressed at low but substantial levels on APMreplete tissues. It has been demonstrated previously by mass spectrometry that not all TAP-independent peptides fail to be presented by APM-replete cells (13). However, the $\mathrm{T}$ cell repertoire against these epitopes derived from ubiquitous housekeeping proteins is thought to be subject to central or peripheral tolerance and therefore does not have the capacity to induce autoimmune tissue damage.

The observation that a 21-mer-long TEIPP prototype peptide presented by DCs was able to activate TEIPP-specific T cells from the TCR-tg mice is highly relevant for the potential use of TEIPP epitopes as a cancer vaccine. Doorduljn and colleagues have also demonstrated in vivo by adoptive transfer that TEIPP-specific $\mathrm{T}$ cells can be efficiently primed by cross-presentation through vaccination with the same long TEIPP peptide and that this can result in activation of cytolytic CD8 ${ }^{+}$TEIPP-specific $\mathrm{T}$ cells (11). This observation gives rise to the key question as to whether vaccine-induced, TEIPP-specific T cells can protect against tumor growth in a therapeutic setting. The original TAP2-deficient RMA-S tumor model that was used initially to define the TEIPP phenomenon was also employed to address this question. This tumor was originally produced in vitro by mutagenesis and was repeatedly selected as an MHC-I ${ }^{\text {lo }}$ variant. Arguably, a tumor line with spontaneous loss of MHC-I or loss of MHC-I subsequent to immunotherapy would be more relevant as a model to evaluate TEIPP therapy of human cancer. Furthermore, as Doorduljn et al. prove that TEIPP-specific T cells are not subjected to central tolerance, a more pronounced therapeutic effect than that observed was expected following vaccination with their prototype TEIPP peptide. A substantial tumor-protective effect was only obtained through combining antitumor vaccination with adoptive transfer of TEIPP-specific $\mathrm{T}$ cells. Nevertheless, these observations may influence cancer immunotherapy, and candidates for human TEIPP epitopes were previously described through the use of herpesvirus-derived TAP inhibitors (14). In the future, we look forward to a better understanding of the human TEIPP repertoire and clinical testing of this intriguing class of antigens for efficacy in immunotherapy of APM-defective human cancers.

\section{Acknowledgments}

Work in the author's laboratory is supported by grants from The Swedish Cancer Society (2013/379), The Cancer Society of Stockholm (144102), and The Swedish Medical Research Council (521-2013-4100), Stockholm City Council Project grant 20140036 (ALF Medicin 2015), the Knut and Alice Wallenberg Foundations, and by support from the Karolinska sponsored Center for Immune Modulatory Therapies for Autoimmunity and Cancer (IMTAC).

Address correspondence to: Rolf Kiessling, Cancer Center Karolinska, R8:01, Karolinska Hospital, S-17176 Stockholm, Sweden, Phone: 46.733428848; E-mail: Rolf.Kiessling@ki.se. 
1. Rosenberg SA. Decade in review-cancer immunotherapy: entering the mainstream of cancer treatment. Nat Rev Clin Oncol. 2014;11(11):630-632.

2. Seliger B, Cabrera T, Garrido F, Ferrone S. HLA class I antigen abnormalities immune escape by malignant cells. Semin Cancer Biol. 2002;12(1):3-13.

3. Kärre K, Ljunggren HG, Piontek G, Kiessling R. Selective rejection of $\mathrm{H}$-2-deficient lymphoma variants suggests alternative immune defence strategy. Nature. 1986;319(6055):675-678.

4. Angell H, Galon J. From the immune contexture to the Immunoscore: the role of prognostic predictive immune markers in cancer. Curr Opin Immunol. 2013;25(2):261-267.

5. Wolpert EZ, et al. Generation of $\mathrm{CD}^{+} \mathrm{T}$ cells specific for transporter associated with antigen processing deficient cells. Proc Natl Acad Sci US A.
1997;94(21):11496-11501.

6. Seidel UJ, Oliveira CC, Lampen MH, van Hall T. A novel category of antigens enabling CTL immunity to tumor escape variants: Cinderella antigens. Cancer Immunol Immunother. 2012;61(1):119-125.

7. van Hall T, et al. Selective cytotoxic T-lymphocyte targeting of tumor immune escape variants. Nat Med. 2006;12(4):417-424.

8. van Hall T, et al. The varicellovirus-encoded TAP inhibitor UL49. J Immunol. 2007;178(2):657-662.

9. Morgan RA, et al. Cancer regression and neurological toxicity following anti-MAGE-A3 TCR gene therapy. J Immunother. 2013;36(2):133-151.

10. Seaman BJ, et al. Audiovestibular dysfunction associated with adoptive cell immunotherapy for melanoma. Otolaryngol Head Neck Surg.
2012;147(4):744-749.

11. Doorduljn EM, et al. TAP-independent self-peptides enhance T cell recognition of immune-escaped tumors. JClin Invest. 2016;126(2):784-794.

12. Carreno BM, et al. Cancer immunotherapy. A dendritic cell vaccine increases the breadth and diversity of melanoma neoantigen-specific T cells. Science. 2015;348(6236):803-808.

13. Weinzierl AO, et al. Features of TAP-independent MHC class I ligands revealed by quantitative mass spectrometry. Eur J Immunol. 2008;38(6):1503-1510.

14. Lampen MH, Verweij MC, Querido B, van der Burg SH, Wiertz EJ, van Hall T. CD8 ${ }^{+}$T cell responses against TAP-inhibited cells are readily detected in the human population. JImmunol. 2010;185(11):6508-6517. 